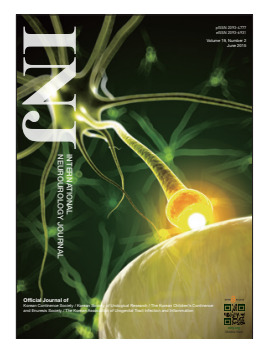

\title{
Advanced Male Urethral and Genital Reconstructive Surgery. 2nd ed.
}

By Steven B. Brandes, Allen F. Morey. New York: Springer/Humana Press; 2013.759 pp. \$219.00. ISBN: 978-1-4614-7707-5

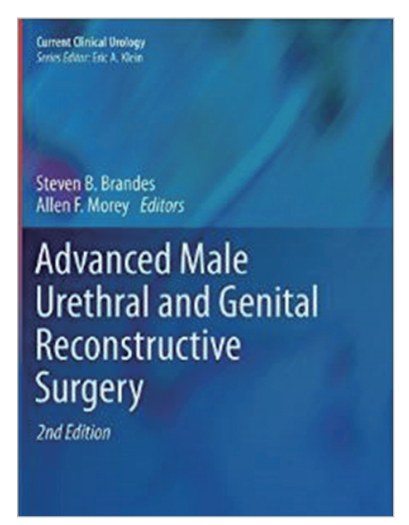

Not all male voiding problems are due to the prostate and one will eventually meet a patient with various urethral conditions including strictures, fistulas, defects and more. The management of these patients can be extremely difficult since no two patients are alike and the presentations are frequently complex. More often than not the surgeon needs to be creative in the operating field and needs to have knowledge of the 'rights' and 'wrongs' in the reconstruction of the urethra and genital tract. In this regard, this book will probably best ful-
Jang Hwan Kim

Department of Urology, Yonsei University College of Medicine
E-mail: jkim@yuhs.ac

fill the needs of anyone planning to surgically attack the problems involving the male urethra. Fifty chapters are dedicated to this subject and explain complex issues such as managing radiation induced urethral stricture, reconstruction of the neophallus urethra, methods of explanting urethral stents, and salvaging failed slings and artificial urinary sphincters. This extensive coverage will no doubt be a valuable resource to not only those entering the reconstruction world but also to those already living in it. 